\title{
Anterior versus Posterior Approach for Ultrasound (US) Guided Shoulder Magnetic Resonance (MR) Arthrograms
}

\author{
Ahmed A. Wahdan ${ }^{1 *}$, Mohamed Refaat', Laila El Kady', Mohamed Mousa', \\ and Ahmed Toreih²
}

${ }^{1}$ Radiology Department, 2Orthopedics Surgery Department, Suez Canal University Hospital, Faculty of Medicine, Suez Canal University, Ismailia Egypt

\begin{abstract}
Background: Shoulder arthrography is an accurate method that can help in diagnosing several diseases. When shoulder arthrography is done combined with magnetic resonance imaging (MRI), a more comprehensive assessment of the cartilaginous structures of the glenohumeral joint is performed. Objective: This study aims to compare the anterior with the posterior ultrasound-guided arthrography injection approaches in achieving optimal needle placement, ensuring the accuracy of shoulder arthrography injections. Subjects and Methods: A total of 38 individuals (suffering from shoulder dislocation) were enrolled, in the study. The efficacy of the technique was evaluated relative to the success or failure of contrast medium administration into the glenohumeral joint, and the number of attempts required to achieve that success. Results: The success rate among the anterior approach group was $84.2 \%$ versus $89.5 \%$ in the posterior approach group. The mean number of trials for the anterior and posterior approaches showed no statistically significant difference while the mean injected volume with the anterior approach was significantly lower than that of the posterior approach group (P-Value: 0.006). Conclusion: The posterior approach has a slight advantage over the anterior approach regarding the injected volume of the contrast medium. However, there were better results yet non-statistically significant regarding the anterior and posterior approaches accuracy, pain tolerance, and the number of trials.
\end{abstract}

Keywords: Shoulder MRA, Ultrasound-guided, Injection, Posterior Approach, Anterior approach, Injection volume.

\section{Introduction}

Magnetic resonance (MR) arthrography is believed to be the gold standard diagnostic technique for evaluation of the glenohumeral joint internal derangement ${ }^{(1)}$. This technique requires the intra-articular injection of contrast material at a certain pressure and volume that are sufficient to separate the anatomical structures of the joint which may cause trauma and harmful effects on the normal anatomical structure of the joint. Meanwhile, this may lead to some difficulties while interpreting the result in addition to serious pain that is experienced by the patient following the procedure. Moreover, radiation is used for fluoroscopic guidance despite the uncertain clinical significance given the quantity of radiation typically utilized ${ }^{(2,3)}$. The real-time ultrasound (US) guided anterior glenohumeral joint injection technique has many 
advantages over the anterior fluoroscopic technique. The anterior fluoroscopic approach is usually applied to infuse either a corticosteroid for frozen shoulder treatment or a contrast agent for computed tomography (CT) or magnetic resonance imaging (MRI) shoulder arthrography(4). Shoulder arthrography is an accurate method that can help in diagnosing several diseases(5). When shoulder arthrography is done combined with magnetic resonance imaging (MRI), a more comprehensive assessment of the cartilaginous structures of the glenohumeral joint is performed ${ }^{(6)}$. Fluoroscopically guided arthrography is less invasive, however, it includes exposure to ionizing radiation in addition to needle malposition risk(5). MRI arthrography following fluoroscopically-guided injection is considered impracticable and time-consuming especially if the MRI machine is far from the fluoroscopy area(7). Meanwhile, the high-resolution US-guided approach for needle placement during shoulder arthrography using the posterior method has been demonstrated by Cicak et al. and is generally applied by orthopedic physicians ${ }^{(8)}$. The practicability of shoulder MR arthrography under ultrasound guidance was assessed using an anterior approach, which is considered to be the conventional radiological method(5). This study aims to compare the anterior with the posterior ultrasound-guided arthrographic injection approach in achieving optimal needle placement, ensuring the accuracy of shoulder arthrography injections.

\section{Patients and Methods}

The study is a comparative cross-sectional analysis. We examined patients with shoulder disorders who have been requested to perform MR-Arthrography and referred to MR-Unit of the radiology department-Suez Canal University Hospital. MR-Arthrography was performed for 19 patients via US guidance by a posterior approach and a similar number of cases was performed with an anterior approach. The efficacy of the technique was evaluated relative to the success or failure of contrast medium administration into the glenohumeral joint, and the number of attempts required to achieve that success.

\section{Ultrasonography guided injection}

In the anterior approach, the patients were lying supine with the shoulder slightly externally rotated. The US. machine, set to muscle-skeleton, was used to visualize the needle. After skin and transducer preparation with alcohol 70\%, the patient's shoulder was draped in a sterile fashion. The needle was inserted at the level of the coracoid, from lateral to medial, aimed at the medial border of the humeral head. The contrast medium can be seen flowing in the direction of the subscapular recess and joint space. The posterior approach was performed using US. machine set to muscle-skeleton, was used to visualize the needle. The patient was either lying obliquely prone on the contralateral shoulder or sitting upright with the back to the radiologist and the ipsilateral hand on the contralateral shoulder. After skin and transducer preparation with alcohol $70 \%$, the patient's shoulder was draped in a sterile fashion. The needle was inserted, from lateral to medial, parallel to the long axis of the transducer, and advanced under US control in the joint between the humeral head and the posterior glenoid labrum.

\section{Statistical analysis}

Because the variables were non-normally nonparametric distributed. Statistical significance was accepted if the P-value was less than 0.05 . The comparisons between the techniques were not adjusted for the patients' sex, age, or right or left shoulder. The distribution of all categorical variables was presented in frequencies. Differences 
in these distributions were tested for statistical significance using chi-square tests. All statistical analyses were performed using SPSS, version 14.0.2 for Windows.

\section{Results}

There was male predilection among our study population as male patients represented about $74 \%$ of the study population compared to female patients (26\%) (figure 1). The mean age of our study population was 32.5 years. Regarding the examined site, there were much more right Sided patients rather than left-sided (21/17), and more male patients than females (28/10) as detailed in a table (1).

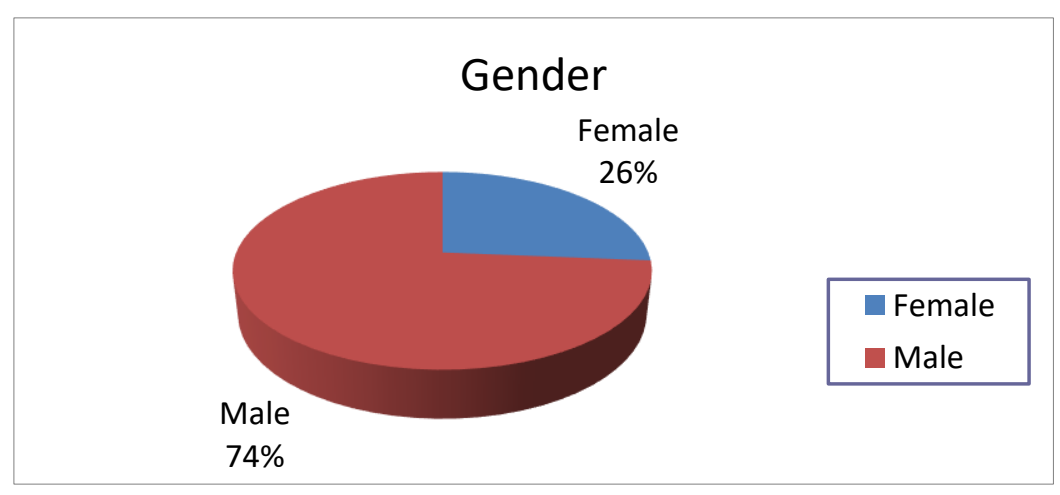

Figure 1: Gender Distribution among the Study Population.

\begin{tabular}{|c|c|c|c|c|c|c|}
\hline \multicolumn{7}{|c|}{ Table 1: Number of Patients, Gender, Age, and Puncture Side per Group } \\
according to Approach of Injection. \\
\hline \multirow{2}{*}{$\begin{array}{c}\text { Approach of } \\
\text { injection }\end{array}$} & $\begin{array}{c}\text { Number of } \\
\text { patients }\end{array}$ & \multicolumn{2}{|c|}{ Gender } & Age & Sidedness \\
\cline { 3 - 8 } & Male & Female & Mean (years) & Right & Left \\
\hline Anterior & 19 & 18 & 1 & 31.9 & 11 & 8 \\
\hline Posterior & 19 & 10 & 9 & 33.1 & 10 & 9 \\
\hline Total & 38 & 28 & 10 & 32.5 & 21 & 17 \\
\hline
\end{tabular}

Among anterior approach group, we had 16 successful injections out of 19 with an $84.2 \%$ success rate, the other failed three patients were switched to the posterior approach and the procedure was successful, so their results were registered with the posterior approach group. Among posterior approach group, we had 17 successful injections out of 19 with $89.5 \%$ success rate, the other two were switched to the anterior approach and the procedure was successful, so their results were registered with the anterior approach group. The mean number of trials for the anterior and posterior approaches was (1.263 and 1.105) respectively showing no statistically significant difference. The mean injected volume with the anterior approach group was $(14.89 \mathrm{ml})$, however, the mean injected volume with the posterior approach group was higher $(17.58 \mathrm{ml})$ showing a statistically significant difference (P-Value: 0.006). The pain tolerability was assessed by verbal expression of the patients either the pain during the injection and intra-articular contrast delivery was tolerable or intolerable, 
$78 \%$ of the anterior approach group gave the comment tolerable, meanwhile, $89 \%$ of the posterior approach group expressed that the pain was tolerable as detailed in table (2).

\section{Discussion}

In relation to the conventional method, arthrographic MRI of the shoulder is believed to increase the diagnostic precision. MRI arthrography is considered to be the most sensitive diagnostic tool used for the detection of shoulder joint disorders ${ }^{(9)}$. By us- ing real-time US, time has been saved more which represents a significant advantage over the fluoroscopy guided arthrography. In addition, no risk for ionizing radiation exposure or radio opaque contrast material infusion is engaged (7). The posterior and/or anterior method for needle position has been demonstrated by many researchers, some were utilizing fluoroscopy while others were applying ultrasound guidance. The posterior approach is applied more frequently by orthopedic specialists, while the anterior approach is considered to be the more conventional approach ${ }^{(5,10)}$.

Table 2: Accuracy, Number of Attempts to Gain Access to the Glenohumeral Joint, Mean Injected Volume, Mean Number of Trials and Pain Tolerance per Group according to Approach of Injection.

\begin{tabular}{|c|c|c|c|c|c|}
\hline & Anterior & Posterior & Total & P-value \\
\hline & Number of patients & 19 & 19 & 38 & - \\
\hline \multicolumn{2}{|l|}{ Successful } & 16 & 17 & 33 & 0.631 \\
\hline \multicolumn{2}{|l|}{ Failed } & 3 & 2 & 5 & 0.630 \\
\hline \multicolumn{2}{|l|}{ Success rate $(\%)$} & $84.2 \%$ & $89.5 \%$ & $86.8 \%$ & 0.523 \\
\hline \multicolumn{2}{|c|}{ Mean injected volume (ml) } & 14.89 & 17.58 & 16.24 & $0.006^{*}$ \\
\hline \multicolumn{2}{|c|}{ Mean number of trials } & 1.263 & 1.105 & 1.184 & 0.293 \\
\hline \multirow{2}{*}{ Tolerance to pain } & Tolerable & 15 & 17 & 32 & \multirow{2}{*}{0.330} \\
\hline & Non-tolerable & 4 & 2 & 6 & \\
\hline
\end{tabular}

$*=p$-value $<0.05$ is significant.

The arthroscopy technique generally follows the posterior route. The path is located between the infraspinatus muscle and the teres minor muscle, with the point of entry being $2 \mathrm{~cm}$ inferior and $1 \mathrm{~cm}$ medial to the posterior angle of the acromion. One probable threat is the injury of the suprascapular nerve or the circumflex scapular vessels. Moreover, if the needle is entered inferior to the teres minor muscle (through the quadrangular space), other possible risks could be involved such as injury of the axillary nerve or the posterior humeral circumflex artery $(5,10)$. In addition, anterior shoulder injections may lead to penetration of the anterior stabilizing structures of the glenohumeral joint ${ }^{(11)}$.
The anterior approach targets the rotator cuff interval and avoids the subscapularis muscle and tendon, inferior glenohumeral ligament, and the antero-inferior labrum of the shoulder. This approach enables the needle insertion into the shoulder until it contacts the medial upper third of the humeral head, just lateral to the joint space, hence also evading the anterosuperior labrum $^{(12)}$. This approach, additionally, avoids the risk of contrast material extravasation, unless the quantity of the injected contrast is exceeding the shoulder joint capacity. If the rupture of the shoulder joint capsule occurs, then, this may lead to extravasation in the subscapularis recess or the biceps tendon sheath but not into the 
subacromial-subdeltoid bursa(13). The standard anterior approach has been showing poor results in successful needle positioning which may therefore leads to preferring the posterior approach in many settings. It was suggested that these approaches should be compared in further studies and that the posterior approach is more favorable ${ }^{(14)}$. In our experience, the posterior approach has slight advantage over the anterior approach, as there was significant statistical difference in the mean injected volume as the mean injected volume with the posterior approach. However, there were better results yet non-statistical significance regarding the anterior and posterior approaches accuracy, pain tolerance and the number of trials.

\section{References}

1. Perdikakis E, Drakonaki E, Maris T, Karantanas $A$. MR arthrography of the shoulder: tolerance evaluation of four different injection techniques. Skeletal Radiol. 2013;42(1):99-105.

2. Larson DB, Rader SB, Forman HP, Fenton LZ. Informing parents about $\mathrm{CT}$ radiation exposure in children: it's OK to tell them. Am J Roentgenol. 2007;189(2):271-5.

3. Messina C, Banfi G, Aliprandi A, Mauri G, Secchi F, Sardanelli F, et al. Ultrasound guidance to perform intra-articular injection of gadolinium-based contrast material for magnetic resonance arthrography as an alternative to fluoroscopy: the time is now. Eur Radiol. 2016;26(5):1221-5.

4. Zwar RB, Read JW, Noakes JB. Sonographically guided glenohumeral joint injection. Am J Roentgenol. 2004;183(1):48-50.

5. Valls R, Melloni P. Sonographic guidance of needle position for $M R$ arthrography of the shoulder. AJR Am J Roentgenol. 1997;169(3):845-7.

6. Jacobson JA, Lin J, Jamadar DA, Hayes CW. Aids to successful shoulder arthrography performed with a fluoroscopically guided anterior approach. Radiographics. 2003;23(2):373-8.

7. Palmer WE. MR arthrography: is it worthwhile? Top Magn Reson imaging TMRI. 1996;8(1):24-43.

8. Ćićak N, Matasović T, Bajraktarević T. Ultrasonographic guidance of needle placement for shoulder arthrography. $J$ ultrasound Med. 1992;11(4):135-7.

9. Lenza $M$, Buchbinder R, Takwoingi $Y$, Johnston R V, Hanchard NCA, Faloppa $F$. Magnetic resonance imaging, magnetic resonance arthrography and ultrasonography for assessing rotator cuff tears in people with shoulder pain for whom surgery is being considered. Cochrane Database Syst Rev. 2013;(9).

10. Tischer T, Vogt S, Kreuz PC, Imhoff AB. Arthroscopic anatomy, variants, and pathologic findings in shoulder instability. Arthrosc J Arthrosc Relat Surg. 2011;27(10):1434-43.

11. Chung CB, Dwek JR, Feng $S$, Resnick D. $M R$ arthrography of the glenohumeral joint: a tailored approach. Am J Roentgenol. 2001;177(1):217-9.

12. Dépelteau H, Bureau NJ, Cardinal E, Aubin B, Brassard P. Arthrography of the shoulder: a simple fluoroscopically guided approach for targeting the rotator cuff interval. Am J Roentgenol. 2004;182(2):329-32.

13. Ehara S, Itoi E, Sashi R. Injection of rotator interval for shoulder arthrography. AJR Am J Roentgenol. 2004;183(4):1172-3.

14. Schumacher Jr HR. Aspiration and injection therapies for joints. Arthritis Care Res (Hoboken). 2003;49(3):41320. 